\title{
Manganese in Black Crusts on Seneca Sandstone
}

\author{
Carol A. Grissom ${ }^{1}$, Edward P. Vicenzi ${ }^{1}$, Richard A. Livingston ${ }^{2}$, Emily Aloiz ${ }^{1}$, Nicole Little ${ }^{1}$, Jennifer \\ Giaccai $^{3}$, and Willa Freedman ${ }^{2}$ \\ ${ }^{1}$ Museum Conservation Institute, Smithsonian Institution, Washington, DC, USA \\ ${ }^{2}$ Materials Science and Engineering, University of Maryland, College Park, MD, USA \\ ${ }^{3}$ Freer/Sackler, Smithsonian Institution, Washington, DC, USA
}

Introduction: Scattered black deposits are found on surfaces of the Smithsonian Castle (1849-1855) and Enid Haupt Garden Gateposts (1987), as seen in Figures 1A. The structures are made of Seneca sandstone, an arkosic micaceous sandstone quarried near Washington, DC. Photographs document that deposits have increased on the relatively new gateposts over the last six years. Generally, black deposits on sandstone are attributed to solubilized silica that incorporates dark particulate material [1]. XRF analysis of a sample taken from a gatepost (Fig. 1B) and in situ analysis of deposits on the Castle instead indicate that the deposits are manganese rich, likely colored by small amounts of manganese oxide. The deposits are thus comparable to desert varnishes on sandstone, which contain mineralized manganese and sometimes iron as a result microbial action [2,3]. Normally soiling on buildings is associated with moisture and rainwater patterns, and the seemingly random locations of the manganese-rich deposits without a clear relationship to water remain a puzzle. Manganese-rich black deposits have not been reported on other sandstone buildings in temperate climates; it is not clear to what extent they are present elsewhere and may have been incorrectly identified. Correct characterization of such deposits is important from a conservation standpoint in order to determine the most effective method of removal, if required.

Results: Cross-sectioned, ground, and polished fragments taken from a gatepost clearly show a black surface region measuring 200-300 $\mathrm{m}$ in thickness using an optical microscope (Fig. 2A). Hyperspectral $\mathrm{X}$-ray imaging in the SEM showed particles in the crust ranging from $22 \%$ manganese at the surface down to $10 \%$ (atomic) in pore spaces (Figs. 2B), as well as minor amounts of iron. Neutron activation analysis (NAA) found only 0.04\% manganese oxide in bulk Seneca sandstone. The small size of the manganese-rich particles $(\sim 100 \mathrm{~nm}$ in diameter), likely to be oxides, and the small number found through the full depth of the visible black area indicates their strong pigmentation properties (Fig. 3A). Multivariate statistical analysis of data collected at $12.5 \mathrm{keV}$ demonstrates that X-ray activation volumes are larger than the particle size (Fig 3B).

Conclusion: The preliminary conclusion is that Mn-enriched crusts on the sandstone are not anthropogenic in origin, but rather mediated by microbial processes such as found in desert varnish. The strong pigmentation and dispersion of particles in pore spaces suggests that it would not be easy to remove the black deposits without loss of stone.

[1] A Milligan, in Stonecleaning, ed. R Webster, (Donhead, London 1992) p. 97-104.

[2] DA McKeown and JE Post, American Mineralogist 86 (2001), p. 701-713. [3]

CM Santelli et al, Geochimica et Cosmochimica Acta 75 (2011), p. 2762-2776. 


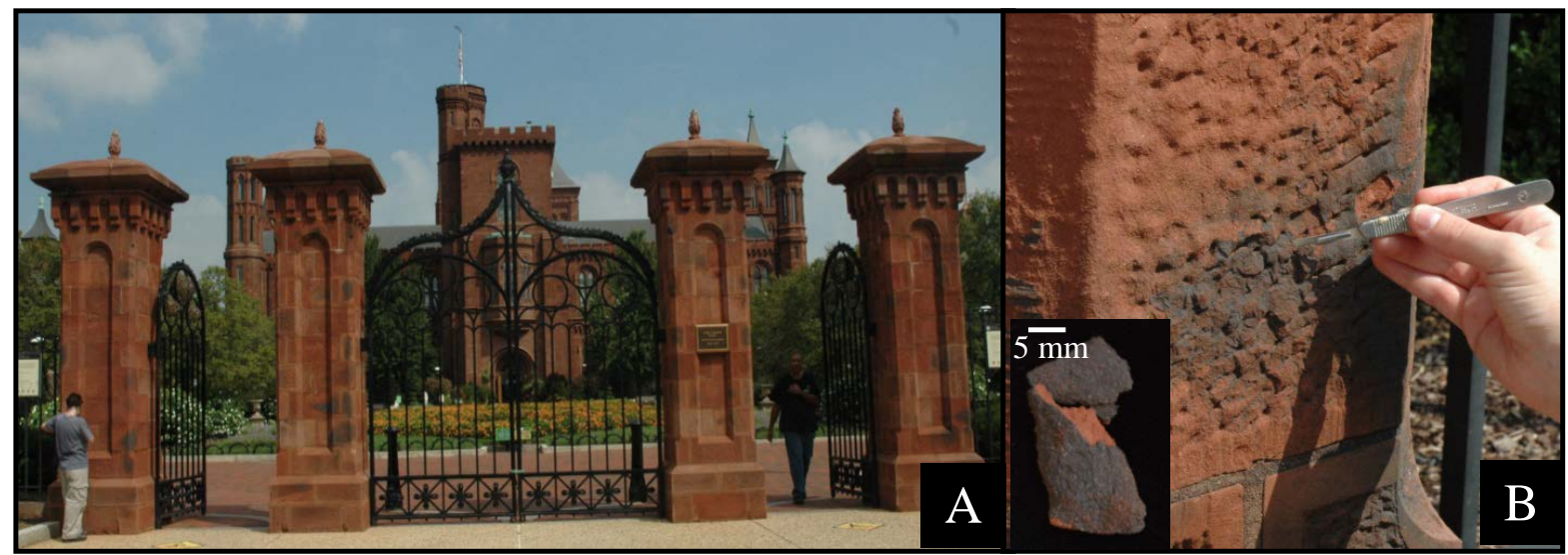

Figure 1 A) Gated entrance to Haupt Garden with Smithsonian Castle in background. B) Sampling black-stained areas on gatepost. Inset: fractured fragment taken from gatepost.

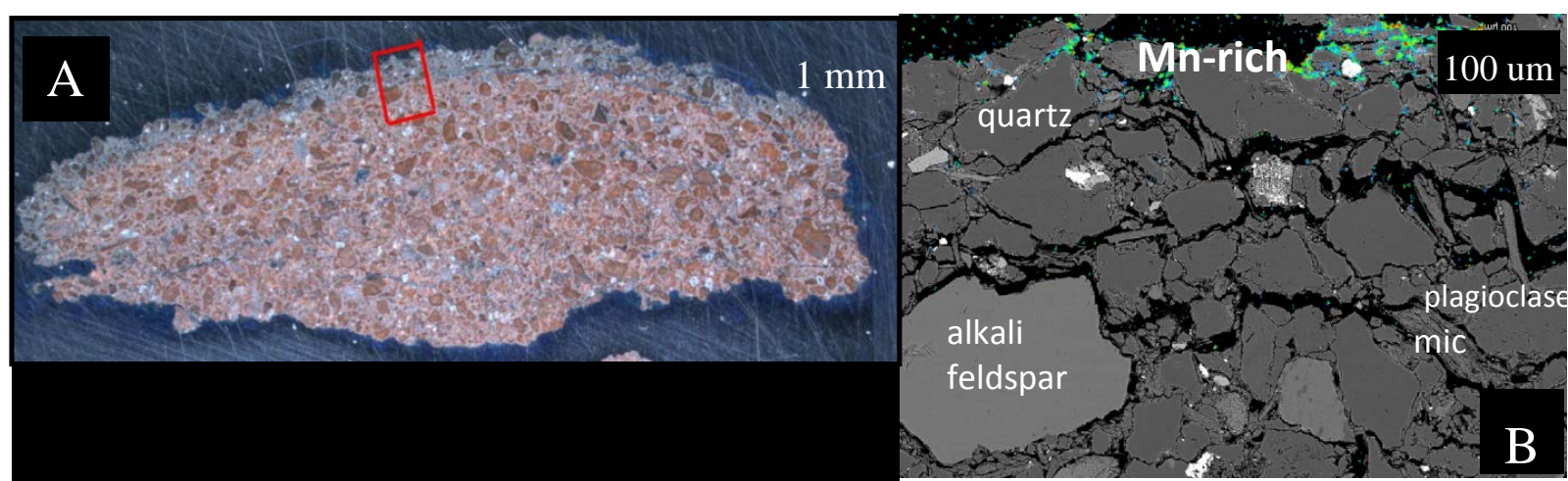

Figure 2 A) Visible light micrograph of polished thick section. B) Quantified Mn map (false colored) superimposed on a BSE image of stone exterior surface region highlighted in A.

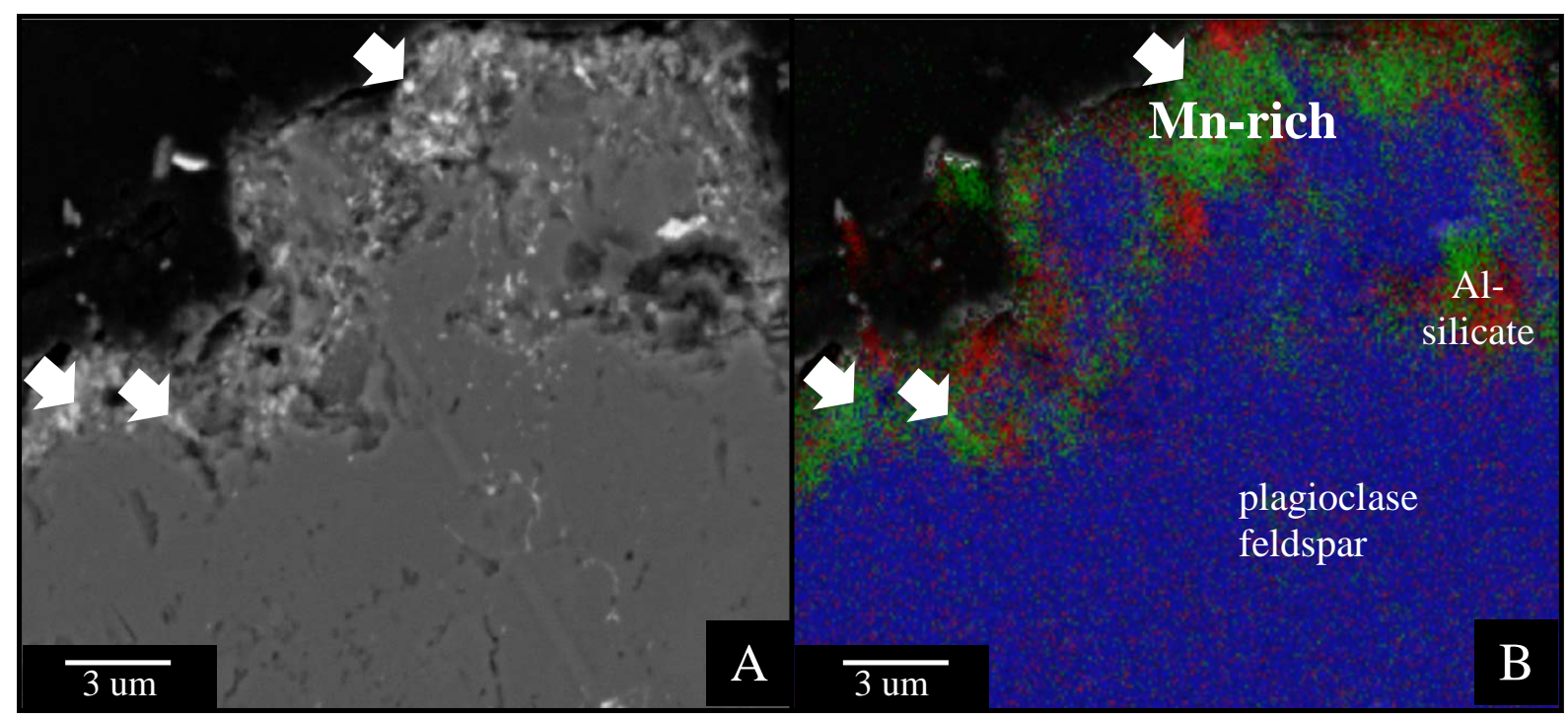

Figure 3 A) Higher magnification BSE image interface showing numerous sub-micron sized high $\mathrm{Z}$ particles at the sandstone-atmosphere interface. B) RGB composite component image derived from hyperspectral X-ray data, where: green-Mn-rich phase; blue-plagioclase feldspar; red-Al silicate. 